NONCOMMUTATIVE HARMONIC ANALYSIS

WITH APPLICATIONS TO PROBABILITY III

BANACH CENTER PUBLICATIONS, VOLUME 96

INSTITUTE OF MATHEMATICS

POLISH ACADEMY OF SCIENCES

WARSZAWA 2012

\title{
FACIAL STRUCTURES OF SEPARABLE AND PPT STATES
}

\author{
SEUNG-HYEOK KYE \\ Department of Mathematics, Seoul National University \\ Seoul 151-742, Korea \\ E-mail: kye@snu.ac.kr
}

\begin{abstract}
A positive semi-definite block matrix (a state if it is normalized) is said to be separable if it is the sum of simple tensors of positive semi-definite matrices. A state is said to be entangled if it is not separable.

It is very difficult to detect the border between separable and entangled states. The PPT (positive partial transpose) criterion tells us that the partial transpose of a separable state is again positive semi-definite, as was observed by M. D. Choi in 1982 from the mathematics side.

In this expository note, we explain the facial structures of the cone of all PPT block matrices, which are naturally characterized by pairs of subspaces of (small) matrices. We also discuss which faces of PPT's induce faces of separables, and which faces of separables are induced by PPT's.
\end{abstract}

1. Introduction. The notion of entanglement originated from quantum mechanics, and has no counterpart in classical mechanics. On the mathematics side, this may be explained by the distinction between commutative order structures and non-commutative order structures: it is well-known that a nonnegative continuous function of two variables is the limit of the sums of nonnegative continuous functions of separable variables. In the language of the tensor products of $C^{*}$-algebras, the positive cone $(C(X) \otimes C(Y))^{+}$of the tensor product of commutative $C^{*}$-algebras $C(X)$ and $C(Y)$ of all continuous functions on compact Hausdorff spaces $X$ and $Y$ coincides with the tensor product $C(X)^{+} \otimes C(Y)^{+}$ of the positive cones.

This is not the case for matrix algebras, the simplest non-commutative case, because the positive cone $\left(M_{n} \otimes M_{m}\right)^{+}$of the tensor product of matrix algebras $M_{n}$ and $M_{m}$ is strictly larger than the tensor product $M_{n}^{+} \otimes M_{m}^{+}$of positive cones $M_{n}^{+}$and $M_{m}^{+}$. Throughout this note, $M_{n}$ denotes the $C^{*}$-algebra of all $n \times n$ matrices with complex

2010 Mathematics Subject Classification: Primary 81P15; Secondary 15A30, 46L05.

Key words and phrases: entanglement, positive partial transpose, positive linear maps, decomposable maps, faces.

The paper is in final form and no version of it will be published elsewhere. 
entries, and $M_{m \times n}$ denotes the inner product space of all $m \times n$ matrices with complex entries.

The notion of entanglement has been studied extensively by quantum physicists since nineties in the relations with possible applications to quantum information theory and quantum computing theory. We refer to the book [3] for related material. Entanglement is a block matrix in $\left(M_{n} \otimes M_{m}\right)^{+}$which does not belong to $M_{n}^{+} \otimes M_{m}^{+}$. One of the main research topics in entanglement theory is to distinguish entanglement from block matrices in $M_{n}^{+} \otimes M_{m}^{+}$, whose elements give rise to separable states.

It is easy to see that if a block matrix gives rise to a separable state then its block transpose is still positive semi-definite. This gives us a necessary condition for separability. The positive semi-definite block matrix with positive semi-definite block transpose is said to be of positive partial transpose (PPT). In this expository note, we explore the boundary structures, or equivalently facial structures of the two cones; the cone consisting of PPT's and the cone consisting of separable states (up to scalar multiple).

Recall that a matrix itself represents a linear functional on the matrix algebra with respect to the Hadamard product. In this correspondence, a positive semi-definite matrix represents a positive linear functional on the matrix algebra, and a density matrix represents a state, a unital positive linear functional on the matrix algebra. Throughout this note, we do not distinguish matrices themselves from linear functionals. In this sense, every element in $M_{n}^{+} \otimes M_{m}^{+}$is a positive linear functional on $M_{n} \otimes M_{m}$, which is separable. We call that just a separable state by abuse of terminology.

The whole structures of operator algebras heavily depend on the order structures, as is seen in the Gelfand-Naimark-Segal representation theorem. In this vein, operator algebraists have studied various types of positive linear maps between operator algebras, and how to distinguish them, since fifties [41, [42. For example, Choi [1] showed that there is a non-decomposable positive linear map between $M_{3}$ by exhibiting an example of a positive semi-definite biquadratic form which is not the sum of squares of bilinear forms. Woronowicz [48] also showed that there exists a non-decomposable positive linear map from $M_{2}$ into $M_{4}$ by exhibiting a special block matrix in $M_{4} \otimes M_{2}$, which is nothing but an example of an entangled state with positive partial transpose. The same thing has been done in $M_{3} \otimes M_{3}$ by Størmer [43]. Recently, many mathematicians are interested in entanglement theory itself. See [1, 2], 28] and [4], for example.

In the second section of this note, we introduce various kinds of entanglement and positive linear maps, and explain dualities between them. In the third section, we characterize faces of various cones introduced in the second section, and explain how to describe faces using the duality. In the last section, we exhibit some examples in the $3 \otimes 3$ case.

Throughout this note, we are concerned with subspaces of the inner product space $M_{m \times n}$ which is inner product space isomorphic to $\mathbb{C}_{n} \otimes \mathbb{C}_{m}$. This is why we do not use the convenient bra-ket notations of physicists, which is natural to describe elements of $\mathbb{C}_{n} \otimes \mathbb{C}_{m}$. Every vector will be considered as a column vector. If $x \in \mathbb{C}^{m}$ and $y \in \mathbb{C}^{n}$ then $x$ will be considered as an $m \times 1$ matrix, and $y^{*}$ will be considered as a $1 \times n$ matrix, and so $x y^{*}$ is an $m \times n$ rank one matrix whose range is generated by $x$ and whose kernel is orthogonal to $y$. For a vector $x$, the notation $\bar{x}$ will be used for the vector whose entries 
are conjugates of the corresponding entries. The notation $\langle\cdot, \cdot\rangle$ will be used for bilinear pairing. For natural numbers $m$ and $n$, we denote by $m \wedge n$ the minimum of $m$ and $n$. Finally, $\left\{e_{i j}: i=1, \ldots, m, j=1, \ldots, n\right\}$ denotes the usual matrix units in $M_{m \times n}$.

2. Entanglement and positive maps. In this section, we will explain what is entanglement and investigate dualities between entanglement and positive linear maps in matrix algebras. Basically, entanglement is a positive semi-definite block matrix in $M_{n} \otimes M_{m}$ which cannot be expressed as the sum of simple tensors of positive semi-positive matrices in $M_{m}$ and $M_{n}$. We also introduce a bilinear pairing between $M_{n} \otimes M_{m}$ and the space $\mathcal{L}\left(M_{m}, M_{n}\right)$ of all linear maps from $M_{m}$ to $M_{n}$. This pairing allows us to understand various cones of block matrices or positive linear maps as dual cones with respect to this pairing.

2.1. Entanglement. For an $m \times n$ matrix $z \in M_{m \times n}$ with the $i$-th row $z_{i}$, we write

$$
\widetilde{z}=\sum_{i=1}^{m} z_{i} \otimes e_{i} \in \mathbb{C}^{n} \otimes \mathbb{C}^{m}
$$

and define the convex cones $\mathbb{V}_{s}$ and $\mathbb{V}^{s}$ in $M_{n} \otimes M_{m}$ by

$$
\begin{aligned}
\mathbb{V}_{s} & =\operatorname{conv}\left\{\widetilde{z} \widetilde{z}^{*} \in M_{n} \otimes M_{m}: \operatorname{rank} z \leq s\right\}, \\
\mathbb{V}^{s} & =\operatorname{conv}\left\{\left(\widetilde{z} \widetilde{z}^{*}\right)^{\tau} \in M_{n} \otimes M_{m}: \operatorname{rank} z \leq s\right\},
\end{aligned}
$$

for $s=1,2, \ldots, m \wedge n$, where

$$
\sum\left(a_{i j} \otimes e_{i j}\right)^{\tau}=\sum a_{j i} \otimes e_{i j}
$$

denotes the block transpose, or partial transpose by the language of quantum physics, and conv $X$ denotes the convex hull generated by $X$.

By a simple calculation, we have

$$
\widetilde{x y^{*}} \widetilde{x y^{*}}{ }^{*}=\bar{y} y^{*} \otimes x x^{*}
$$

for $x \in \mathbb{C}^{m}$ and $y \in \mathbb{C}^{n}$, and so it follows that

$$
\mathbb{V}_{1}=M_{n}^{+} \otimes M_{m}^{+}=\mathbb{V}^{1}
$$

We also have the following chain of inclusions;

$$
\begin{aligned}
& \mathbb{V}_{1} \subset \mathbb{V}_{2} \subset \ldots \subset \mathbb{V}_{m \wedge n}=\left(M_{n} \otimes M_{m}\right)^{+}, \\
& \mathbb{V}^{1} \subset \mathbb{V}^{2} \subset \ldots \subset \mathbb{V}^{m \wedge n} .
\end{aligned}
$$

We say that a positive semi-definite block matrix $A \in\left(M_{n} \otimes M_{m}\right)^{+}$is separable if $A \in \mathbb{V}_{1}$, and entangled if it is not separable. A block matrix in $\mathbb{V}_{s} \backslash \mathbb{V}_{s-1}$ is said to be of Schmidt number s. See [0].

For example, the matrix

$$
\left(\begin{array}{llll}
1 & 0 & 1 & 0 \\
0 & 0 & 0 & 0 \\
1 & 0 & 1 & 0 \\
0 & 0 & 0 & 0
\end{array}\right)
$$


is separable in $M_{2} \otimes M_{2}$ since it is $\widetilde{z} \widetilde{z}^{*}$ with the rank one matrix $z=\left(\begin{array}{ll}1 & 0 \\ 1 & 0\end{array}\right)$. On the other hand, the rank one matrix

$$
A=\left(\begin{array}{llll}
1 & 0 & 0 & 1 \\
0 & 0 & 0 & 0 \\
0 & 0 & 0 & 0 \\
1 & 0 & 0 & 1
\end{array}\right)
$$

is entangled, since it is $\widetilde{z} \widetilde{z}^{*}$ with the rank two matrix $z=\left(\begin{array}{ll}1 & 0 \\ 0 & 1\end{array}\right)$. If a positive semidefinite matrix $A$ is not of rank one, then it is extremely difficult to determine whether it is separable or entangled. Note that the block transpose

$$
A^{\tau}=\left(\begin{array}{llll}
1 & 0 & 0 & 0 \\
0 & 0 & 1 & 0 \\
0 & 1 & 0 & 0 \\
0 & 0 & 0 & 1
\end{array}\right)
$$

of $A$ in $M_{2} \otimes M_{2}$ is not positive semi-definite.

2.2. Positive partial transpose. If $z=x y^{*}$ is a rank one matrix with column vectors $x$ and $y$, then

$$
\left(\widetilde{z} \widetilde{z}^{*}\right)^{\tau}=\widetilde{w} \widetilde{w}^{*}, \quad \text { with } w=\bar{x} y^{*},
$$

is positive semi-definite by a direct simple calculation. So, we have

$$
\mathbb{V}_{1} \subseteq \mathbb{V}_{m \wedge n} \cap \mathbb{V}^{m \wedge n},
$$

to get a necessary condition for separability, which is called the PPT (positive partial transpose) criterion by quantum physicists. See [12] and 38].

We define

$$
\mathbb{T}=\mathbb{V}_{m \wedge n} \cap \mathbb{V}^{m \wedge n} .
$$

A positive semi-definite block matrix is said to be of positive partial transpose (PPT) if it belongs to $\mathbb{T}$. The PPT criterion says that if a positive semi-definite block matrix is separable then it is of PPT. In the case of $M_{2} \otimes M_{n}$, it was shown by Woronowicz [48] that the converse holds if and only if $n=2$ or 3 . In other words, $\mathbb{T}=\mathbb{V}_{1}$ in $M_{2} \otimes M_{n}$ if and only if $n \leq 3$. Choi [12] also gave an example of a non-separable PPT matrix in $M_{3} \otimes M_{3}$. Many examples of PPT entangled states have been found by quantum physicists. See [4, [5], 14], 15], 25], 26] and 40], for examples.

2.3. Positive linear maps. A linear map $\phi: M_{m} \rightarrow M_{n}$ is said to be positive if $\phi\left(M_{m}^{+}\right) \subset M_{n}^{+}$. It is said to be s-positive if $\phi_{s}: M_{s}\left(M_{m}\right) \rightarrow M_{s}\left(M_{n}\right)$ is positive, where

$$
\phi_{s}:\left[x_{i j}\right] \mapsto\left[\phi\left(x_{i j}\right)\right],
$$

and $s$-copositive if $\phi^{s}: M_{s}\left(M_{m}\right) \rightarrow M_{s}\left(M_{n}\right)$ is positive, where

$$
\phi^{s}:\left[x_{i j}\right] \mapsto\left[\phi\left(x_{j i}\right)\right] .
$$

We denote by $\mathbb{P}_{s}$ (respectively $\mathbb{P}^{s}$ ) the convex cone of all $s$-positive linear maps (respectively $s$-copositive linear maps). The transpose map t $: M_{n} \rightarrow M_{n}$ is a typical example of 
positive linear map which is not 2-positive. A linear map is said to be completely positive (respectively completely copositive) if it is $s$-positive (respectively $s$-copositive) for every $s=1,2, \ldots$. The following is very useful to deal with completely positive linear maps between matrix algebras. See [10] and [31].

THEOREM 2.1. For a linear map $\phi: M_{m} \rightarrow M_{n}$, the following are equivalent:

(i) $\phi$ is completely positive,

(ii) the matrix

$$
\left[\phi\left(e_{i j}\right)\right]=\left(\begin{array}{ccc}
\phi\left(e_{11}\right) & \cdots & \phi\left(e_{1 n}\right) \\
\vdots & \ddots & \vdots \\
\phi\left(e_{m 1}\right) & \cdots & \phi\left(e_{m m)}\right.
\end{array}\right)
$$

in $M_{m}\left(M_{n}\right)=M_{n} \otimes M_{m}$ is positive semi-definite,

(iii) $\phi$ is $(m \wedge n)$-positive,

(iv) $\phi$ is of the form

$$
\phi_{\mathcal{V}}=\sum_{V \in \mathcal{V}} \phi_{V}
$$

where $\phi_{V}: X \mapsto V^{*} X V$ for $V \in M_{m \times n}$ and $\mathcal{V}$ is a subset of $M_{m \times n}$.

A similar characterization for completely copositive linear maps also holds with the maps

$$
\phi^{\mathcal{V}}=\sum_{V \in \mathcal{V}} \phi^{V}
$$

where $\phi^{V}: X \mapsto V^{*} X^{\mathrm{t}} V$ for $V \in M_{m \times n}$.

A positive linear map $\phi: M_{m} \rightarrow M_{n}$ is said to be decomposable if it is the sum of a completely positive linear map and a completely copositive linear map. We denote by $\mathbb{D}$ the convex cone of all decomposable positive linear maps, that is,

$$
\mathbb{D}=\mathbb{P}_{m \wedge n}+\mathbb{P}^{m \wedge n} \text {. }
$$

There are many examples of indecomposable positive linear maps in the literature. See [7], [1], 17, 18, [19, 29], 32, 37, 39], 43], 45] and [46, for examples. Especially, examples of indecomposable positive linear maps were constructed in [47] using PPT entangled states.

2.4. Dualities. For a block matrix $A=\sum_{i, j=1}^{m} a_{i j} \otimes e_{i j} \in M_{n} \otimes M_{m}$ and a linear map $\phi \in \mathcal{L}\left(M_{m}, M_{n}\right)$, we define the bilinear pairing by

$$
\langle A, \phi\rangle=\operatorname{Tr}\left[\left(\sum_{i, j=1}^{m} \phi\left(e_{i j}\right) \otimes e_{i j}\right) A^{\mathrm{t}}\right]=\sum_{i, j=1}^{m}\left\langle\phi\left(e_{i j}\right), a_{i j}\right\rangle .
$$

Then the pair $(\mathbb{T}, \mathbb{D})$ is dual in the sense

$$
\begin{aligned}
& \phi \in \mathbb{D} \Longleftrightarrow\langle A, \phi\rangle \geq 0 \text { for every } A \in \mathbb{T}, \\
& A \in \mathbb{T} \Longleftrightarrow\langle A, \phi\rangle \geq 0 \text { for every } \phi \in \mathbb{D},
\end{aligned}
$$

and similarly for the pairs

$$
\left(\mathbb{V}_{s}, \mathbb{P}_{s}\right), \quad\left(\mathbb{V}^{s}, \mathbb{P}^{s}\right)
$$


See [16]. We may summarize these dualities as follows:

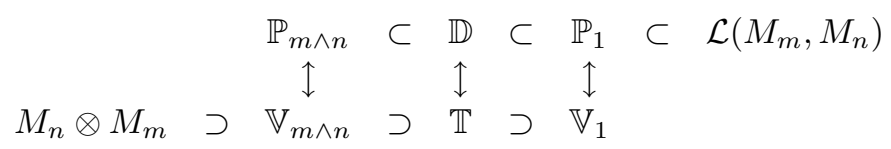

These dualities are very useful to detect exposed faces in various situations, and used to describe maximal faces of $\mathbb{P}_{1}\left[33\right.$. By the duality of the pair $\left(\mathbb{V}_{1}, \mathbb{P}_{1}\right)$, it is also possible to detect entanglement using a positive linear map. More precisely, a positive semi-definite block matrix $A$ in $M_{n} \otimes M_{m}$ is entangled if and only if there exists a positive linear map $\phi$ such that $\langle A, \phi\rangle<0$. This linear map $\phi$ is called an entanglement witness by quantum physicists. See [24].

3. Faces. In this section, we describe facial structures of the various cones introduced in the previous section, and how they are related with respect to the dualities. In the case of the cone $\mathbb{D}$ of all decomposable maps and $\mathbb{T}$ of all PPT's, it turns out that every face is determined by a pair of subspaces of $m \times n$ matrices. For the case of the cone $\mathbb{V}_{1}$ of separable states, we characterize which faces are induced by the larger cone $\mathbb{T}$.

3.1. Faces for completely positive maps. Recall that every element of $\mathbb{P}_{m \wedge n}$ (respectively $\mathbb{P}^{m \wedge n}$ ) is of the form

$$
\phi_{\mathcal{V}}: X \mapsto \sum_{i=1}^{\nu} V_{i}^{*} X V_{i} \quad\left(\text { respectively } \phi^{\mathcal{V}}: X \mapsto \sum_{i=1}^{\nu} V_{i}^{*} X^{\mathrm{t}} V_{i}\right),
$$

where $\mathcal{V}=\left\{V_{1}, \ldots, V_{\nu}\right\} \subset M_{m \times n}$. For a given subspace $E$ of $M_{m \times n}$, we define

$$
\begin{aligned}
& \Phi_{E}=\left\{\phi_{\mathcal{V}} \in \mathbb{P}_{m \wedge n}: \operatorname{span} \mathcal{V} \subset E\right\}, \\
& \Phi^{E}=\left\{\phi^{\mathcal{V}} \in \mathbb{P}^{m \wedge n}: \operatorname{span} \mathcal{V} \subset E\right\} .
\end{aligned}
$$

Then the correspondence $E \mapsto \Phi_{E}$ (respectively $E \mapsto \Phi^{E}$ ) is a lattice isomorphism from the lattice of all subspaces of $M_{m \times n}$ onto the lattice of all faces of $\mathbb{P}_{m \wedge n}$ (respectively $\left.\mathbb{P}^{m \wedge n}\right)$ 34.

3.2. Faces for decomposable maps. Therefore, it is easy to see that every face of the cone

$$
\mathbb{D}=\operatorname{conv}\left(\mathbb{P}_{m \wedge n}, \mathbb{P}^{m \wedge n}\right)
$$

is of the form

$$
\sigma(D, E):=\operatorname{conv}\left(\Phi_{D}, \Phi^{E}\right)
$$

for a pair $(D, E)$ of subspaces of $M_{m \times n}$. This pair is uniquely determined under the assumption

$$
\sigma(D, E) \cap \mathbb{P}_{m \wedge n}=\Phi_{D}, \quad \sigma(D, E) \cap \mathbb{P}^{m \wedge n}=\Phi^{E} .
$$

See [36]. It is very difficult in general to determine what kinds of pairs $(D, E)$ give rise to faces of $\mathbb{D}$. In the case of $m=n=2$, we found all faces in terms of pairs of subspaces [6]. See also 35 . 
3.3. Faces for positive partial transpose. It is well-known that every face of $\mathbb{V}_{m \wedge n}=$ $\left(M_{n} \otimes M_{m}\right)^{+}$is of the form

$$
\Psi_{D}=\left\{A \in\left(M_{n} \otimes M_{m}\right)^{+}: \mathcal{R} A \subset \widetilde{D}\right\}
$$

for a subspace $D$ of $M_{m \times n}$, where $\mathcal{R} A$ is the range space of $A$ and $\widetilde{D}=\left\{\widetilde{z}: \mathbb{C}^{n} \otimes \mathbb{C}^{m}\right.$ : $z \in D\}$. On the other hand, every face of $\mathbb{V}^{m \wedge n}$ is of the form

$$
\Psi^{E}=\left\{A \in M_{n} \otimes M_{m}: A^{\tau} \in \Psi_{E}\right\}
$$

for a subspace $E$ of $M_{m \times n}$. Therefore, every face of $\mathbb{T}=\mathbb{V}_{m \wedge n} \cap \mathbb{V}^{m \wedge n}$ is of the form

$$
\tau(D, E):=\Psi_{D} \cap \Psi^{E}
$$

for a pair $(D, E)$ of subspaces of $M_{m \times n}$. This pair is determined uniquely under the assumption

$$
\operatorname{int} \tau(D, E) \subset \operatorname{int} \Psi_{D}, \quad \operatorname{int} \tau(D, E) \subset \operatorname{int} \Psi^{E}
$$

3.4. Duality of faces. Let $C_{1}$ and $C_{2}$ be convex cones which are dual with respect to the bilinear pairing $\langle\cdot, \cdot\rangle$ as in the case of $\mathbb{D}$ and $\mathbb{T}$. For a subset $S$ of $C_{1}$, the set

$$
S^{\prime}=\left\{y \in C_{2}:\langle x, y\rangle=0 \text { for each } x \in S\right\}
$$

is an exposed face of $C_{2}$. In the dual convex cones $\mathbb{D}$ and $\mathbb{T}$, it is easy to see that

$$
\tau(D, E)^{\prime}=\sigma\left(D^{\perp}, E^{\perp}\right)
$$

gives rise to an exposed face of $\mathbb{D}$. It should be noted that not every face of $\mathbb{D}$ arises in this way even in the simplest case of $m=n=2$. See [6]. Nevertheless, every face of the cone $\mathbb{T}$ arises from this duality. More precisely, it was shown in [21] that every face of the cone $\mathbb{T}$ is of the form

$$
\sigma(D, E)^{\prime}:=\{A \in \mathbb{T}:\langle A, \phi\rangle=0 \text { for every } \phi \in \sigma(D, E)\}=\tau\left(D^{\perp}, E^{\perp}\right)
$$

for a face $\sigma(D, E)$ of the cone $\mathbb{D}$.

With the information in [6], we can list up all pairs $(D, E)$ of subspaces which give rise to faces of $\mathbb{T}$ in the case of $M_{2} \otimes M_{2}$ as follows:

$$
\begin{aligned}
& (1,1): D=\mathbb{C} x y^{*}, E=\mathbb{C} \bar{x} y^{*} \\
& (2,2): D=\operatorname{span}\left\{x y^{*}, z w^{*}\right\}, E=\operatorname{span}\left\{\bar{x} y^{*}, \bar{z} w^{*}\right\} \quad(\text { where } x \nVdash z \text { or } y \nVdash w) \\
& (3,3): D=\left\{x y^{*}\right\}^{\perp}, E=\left\{\bar{x} y^{*}\right\}^{\perp} \\
& (3,3): D=V^{\perp}, E=W^{\perp} \\
& (3,4): D=V^{\perp}, E=M_{2 \times 2} \\
& (4,3): D=M_{2 \times 2}, E=W^{\perp} \\
& (4,4): D=M_{2 \times 2}, E=M_{2 \times 2},
\end{aligned}
$$

where $(s, t)$ in the first column denotes the dimensions of $D$ and $E$ respectively, and $x \| y$ means that $x$ is parallel to $y$. In the case of $(3,3), V$ and $W$ are rank two matrices with nonzero $y_{0}, y_{1}, y_{2} \in \mathbb{C}^{2}$ such that $V y_{i} \| \overline{W y_{i}}$ for each $i=0,1,2$ and $y_{i} \nVdash y_{j}$ for $i \neq j$. In the cases of $(3,4)$ and $(4,3), V$ and $W$ are arbitrary rank two matrices. 
Since $\mathbb{T}=\mathbb{V}_{1}$ in the case of $M_{2} \otimes M_{2}$, this gives us the complete list of faces of the cone $\mathbb{V}_{1}$ of all separable positive semi-definite block matrices in $M_{2} \otimes M_{2}$ in terms of pairs of subspaces of $M_{2 \times 2}$.

3.5. Faces for separable states. Let $C_{1} \subset C_{2}$ be convex sets. A face $F_{1}$ of $C_{1}$ is induced by a face of $C_{2}$ if it is of the form $F_{1}=C_{1} \cap F_{2}$ for a face $F_{2}$ of $C_{2}$. In this case, $F_{2}$ is uniquely determined under the assumption $\operatorname{int} F_{1} \subset \operatorname{int} F_{2}$. We says that $F_{1}$ is induced by $F_{2}$, or $F_{2}$ induces $F_{1}$ if

$$
F_{1}=C_{1} \cap F_{2}, \quad \text { int } F_{1} \subset \operatorname{int} F_{2} .
$$

The next theorem [8] characterizes faces of $\mathbb{T}$ which induce faces of $\mathbb{V}_{1}$. This also implies the range criterion for separability [26].

TheOREm 3.1. Let $(D, E)$ be a pair of subspaces of $M_{m \times n}$. Then the following are equivalent:

(i) The pair $(D, E)$ gives rise to a nontrivial face $\tau(D, E)$ of $\mathbb{T}$ which induces a face of $\mathbb{V}_{1}$.

(ii) There exist $x_{1}, \ldots, x_{\alpha} \in \mathbb{C}^{m}$ and $y_{1}, \ldots, y_{\alpha} \in \mathbb{C}^{n}$ such that

$$
D=\operatorname{span}\left\{x_{1} y_{1}^{*}, \ldots, x_{\alpha} y_{\alpha}^{*}\right\}, \quad E=\operatorname{span}\left\{\overline{x_{1}} y_{1}^{*}, \ldots, \overline{x_{\alpha}} y_{\alpha}^{*}\right\} .
$$

In the case of $M_{2} \otimes M_{2}$, we note that possible pairs of dimensions of subspaces satisfying the conditions in the Theorem 3.1 are

$$
(1,1), \quad(2,2), \quad(3,3), \quad(3,4), \quad(4,3), \quad(4,4),
$$

as we have already seen in the previous subsection. In the case of $M_{2} \otimes M_{n}$, possible pairs of dimensions of subspaces satisfying the conditions are as follows:

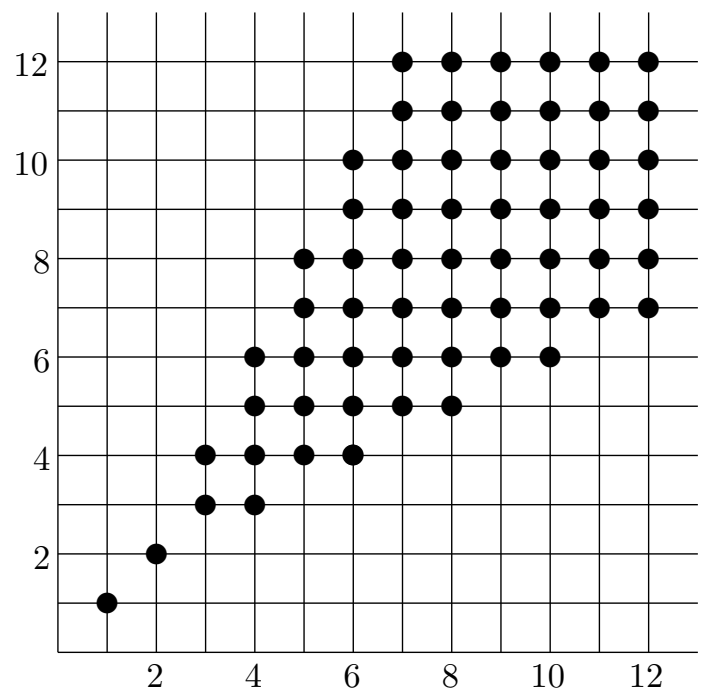

Next, we characterize faces of $\mathbb{V}_{1}$ which are induced by faces of $\mathbb{T}$. For a face $F$ of $\mathbb{V}_{1}$, we denote by $R_{F}$ the set of all $m \times n$ rank one matrices $z$ such that $\widetilde{z} \widetilde{z}^{*} \in F$, which generate extremal rays of the cone $F$. If $F$ is induced by $\tau(D, E)$ then we have

$$
D=\operatorname{span} R_{F}, \quad E=\operatorname{span} R_{F^{\tau}},
$$


where $F^{\tau}=\left\{A^{\tau}: A \in F\right\}$.

TheOREM 3.2. For a face $F$ of $\mathbb{V}_{1}$, the following are equivalent:

(i) The face $F$ of $\mathbb{V}_{1}$ is induced by a face of $\mathbb{T}$.

(ii) If $x y^{*}$ is a rank one matrix in $\left(\operatorname{span} R_{F}\right) \backslash R_{F}$ then $x y^{*} \notin \operatorname{span} R_{F^{\tau}}$.

4. Examples. Two convex cones $\mathbb{V}_{1} \subset \mathbb{T}$ in $M_{n} \otimes M_{m}$ share faces with each other in various sense. Some convex cones are themselves faces of both cones. For example, the face $\tau(D, E)$ of $\mathbb{T}$ is also a face of $\mathbb{V}_{1}$ in itself whenever $\operatorname{dim} D$ or $\operatorname{dim} E$ is less than or equal to $m \wedge n$ by [27].

Some faces of a cone induce faces of the other cone, or are induced by faces of the other cone. Some faces of the cones are independent of the other cones. In this section, we give examples of that last kind of faces of $\mathbb{T}$ and $\mathbb{V}_{1}$. Faces of $\mathbb{T}$ which are independent of $\mathbb{V}_{1}$ give rise to the notion of 'edge states'. A PPT entangled state $A$ in $\mathbb{T} \backslash \mathbb{V}_{1}$ is called a PPT entangled edge state if the proper face of $\mathbb{T}$ containing $A$ as an interior point does not contain a separable state. We call that just an entangled edge state in this note.

Let $\sigma(D, E)$ be a proper face of the cone $\mathbb{D}$. Then we have the following two cases:

$$
\operatorname{int} \sigma(D, E) \subset \operatorname{int} \mathbb{P}_{1} \quad \text { or } \quad \sigma(D, E) \subset \partial \mathbb{P}_{1},
$$

since $\sigma(D, E)$ is a convex subset of the cone $\mathbb{P}_{1}$, where $\partial C=C \backslash \operatorname{int} C$ denotes the boundary of the convex set $C$. We have shown in [21, [23] that

$$
\operatorname{int} \sigma(D, E) \subset \operatorname{int} \mathbb{P}_{1} \Longleftrightarrow \sigma(D, E)^{\prime} \cap \mathbb{V}_{1}=\{0\} .
$$

Therefore, we see that if $\sigma(D, E)$ is a face of $\mathbb{D}$ with $\operatorname{int} \sigma(D, E) \subset \operatorname{int} \mathbb{P}_{1}$ then every nonzero element in the dual face $\sigma(D, E)^{\prime}$ gives rise to an entangled edge state up to constant multiplications. Furthermore, every entangled edge state arises in this way, since every face of the cone $\mathbb{T}$ arises from the duality, by the relation (1).

4.1. Generalized Choi maps. We begin with the map $\Phi[a, b, c]: M_{3} \rightarrow M_{3}$ defined by

$$
\Phi[a, b, c]: x \mapsto\left(\begin{array}{ccc}
a x_{11}+b x_{22}+c x_{33} & 0 & 0 \\
0 & a x_{22}+b x_{33}+c x_{11} & 0 \\
0 & 0 & a x_{33}+b x_{11}+c x_{22}
\end{array}\right)-x
$$

for $x=\left(x_{i j}\right) \in M_{3}$, as was studied in [7]. Recall that $\Phi[2,2,2]$ is the first example [9] of a 2-positive linear map which is not 3-positive, and $\Phi[2,0,1]$ is an example [13] of an extremal positive linear map which is not decomposable. It was shown that $\Phi[a, b, c]$ is positive if and only if

$$
a \geq 1, \quad a+b+c \geq 3, \quad 1 \leq a \leq 2 \Longrightarrow b c \geq(2-a)^{2},
$$

and decomposable if and only if

$$
a \geq 1, \quad 1 \leq a \leq 3 \Longrightarrow b c \geq\left(\frac{3-a}{2}\right)^{2} .
$$

Therefore, every $\Phi[a, b, c]$ with the condition

$$
1<a<3, \quad 4 b c=(3-a)^{2}
$$


gives rise to an element of $\partial \mathbb{D} \cap \operatorname{int} \mathbb{P}_{1}$, whenever $b \neq c$. Furthermore, we have a decomposition

$$
\Phi[a, b, c]=\frac{a-1}{2} \Phi[3,0,0]+\frac{3-a}{2} \Phi\left[1, \sqrt{\frac{b}{c}}, \sqrt{\frac{c}{b}}\right]
$$

into the sum of a completely positive map and a completely copositive map. If we fix $b$ and $c$, then we see that the family $\{\Phi[a, b, c]: 1 \leq a \leq 3\}$ is a line segment, and so it suffices to consider the map $\Phi[2, b, c]$. We also see that

$$
\begin{gathered}
\Phi[3,0,0]=\phi_{e_{11}-e_{22}}+\phi_{e_{22}-e_{33}}+\phi_{e_{33}-e_{11}}, \\
\Phi\left[1, \sqrt{\frac{b}{c}}, \sqrt{\frac{c}{b}}\right]=\phi^{\mu e_{12}-\lambda e_{21}}+\phi^{\mu e_{23}-\lambda e_{32}}+\phi^{\mu e_{31}-\lambda e_{13}},
\end{gathered}
$$

where $\lambda=\left(\frac{b}{c}\right)^{1 / 4}$ and $\mu=\left(\frac{c}{b}\right)^{1 / 4}$, and so $\lambda \mu=1$ and $\lambda \neq 1$.

4.2. Construction of PPTES with dualities. We denote by $\tau(D, E)$ the dual face of $\mathbb{T}$ determined by $\Phi[a, b, c]$ in $(3)$ by the relation (1). Then we see that

$$
\begin{aligned}
D & =\left\{e_{11}-e_{22}, e_{22}-e_{33}, e_{33}-e_{11}\right\}^{\perp} \\
& =\operatorname{span}\left\{e_{12}, e_{21}, e_{23}, e_{32}, e_{31}, e_{13}, e_{11}+e_{22}+e_{33}\right\}, \\
E & =\left\{\mu e_{12}-\lambda e_{21}, \mu e_{23}-\lambda e_{32}, \mu e_{31}-\lambda e_{13}\right\}^{\perp} \\
& =\operatorname{span}\left\{\lambda e_{12}+\mu e_{21}, \lambda e_{23}+\mu e_{32}, \lambda e_{31}+\mu e_{13}, e_{11}, e_{22}, e_{33}\right\},
\end{aligned}
$$

where $\lambda \mu=1$ and $\lambda \neq \pm 1$, as before. We see that every nonzero block matrix in $\tau(D, E)$ gives rise to an entangled edge state by (2). Note that

$$
\operatorname{dim} D=7, \quad \operatorname{dim} E=6 .
$$

Entangled edge states may be classified by their range dimensions as was studied in [40. An entangled edge state $A$ is said to be of type $(s, t)$, or an $(s, t)$-edge state if the range dimension of $A$ is $s$ and the range dimension of $A^{\tau}$ is $t$. By careful choices of block matrices in $\tau(D, E)$, it is possible [22, 23] to find following types

$$
(4,4), \quad(6,5), \quad(7,5), \quad(7,6)
$$

of entangled edge states in $M_{3} \otimes M_{3}$. It is also possible [22] to modify the above construction to find $(8,5)$-entangled edge states.

Another method to construct $(4,4)$-entangled edge states is to use the notion of unextendable product bases [4], 15]. These examples arise from 4-dimensional subspaces of $M_{3}$ which have no rank one matrices and whose orthogonal complements have orthonormal bases consisting of rank one matrices. On the other hand, the construction explained above arises from 4-dimensional subspaces of $M_{3}$ which have no rank one matrices and whose orthogonal complements have six rank one matrices up to constant multiples. Professor Young-Hoon Kiem informed the author that the latter is the generic case for 4-dimensional subspaces of $M_{3}$. In other words, every generic 4-dimensional subspace of $M_{3}$, in algebraic geometric sense, has no rank one matrices and its orthogonal complement has six rank one matrices up to constant multiples.

Entangled edge states of types $(5,5)$ and $(6,6)$ were also found by Clarisse [14] and Ha [20], which turned out 30] to be extremal in the cone $\mathbb{T}$, as in the cases of $(4,4)$-entangled edge states. 
4.3. A face of separable states which is not induced by PPT. Consider the Choi map $\Phi[2,0,1]$ which is an indecomposable positive linear map in $M_{3}$ and generates an extremal ray of the cone $\mathbb{P}_{1}$ :

$$
\phi:\left[a_{i j}\right] \mapsto\left[\begin{array}{ccc}
a_{11}+a_{33} & -a_{12} & -a_{13} \\
-a_{21} & a_{22}+a_{11} & -a_{23} \\
-a_{31} & -a_{32} & a_{33}+a_{22}
\end{array}\right] .
$$

Let $F$ be the dual face of $\mathbb{V}_{1}$ given by this map, that is,

$$
F=\left\{A \in \mathbb{V}_{1}:\langle A, \phi\rangle=0\right\} .
$$

Then the set $R_{F}$ of all $3 \times 3$ rank one matrices $z$ such that $\widetilde{z} \widetilde{z}^{*} \in F$ consists of the following rank one matrices:

$$
\left(\begin{array}{lll}
0 & 0 & 1 \\
0 & 0 & 0 \\
0 & 0 & 0
\end{array}\right), \quad\left(\begin{array}{lll}
0 & 0 & 0 \\
1 & 0 & 0 \\
0 & 0 & 0
\end{array}\right), \quad\left(\begin{array}{lll}
0 & 0 & 0 \\
0 & 0 & 0 \\
0 & 1 & 0
\end{array}\right), \quad\left(\begin{array}{ccc}
1 & \alpha & \bar{\gamma} \\
\bar{\alpha} & 1 & \beta \\
\gamma & \bar{\beta} & 1
\end{array}\right)
$$

where $|\alpha|=|\beta|=|\gamma|=\alpha \beta \gamma=1$. Note that

$$
\operatorname{span} R_{F}=\left\{\left[a_{i j}\right] \in M_{3}: a_{11}=a_{22}=a_{33}\right\}
$$

is a 7 -dimensional subspace of $M_{3 \times 3}$. We also note that a rank one matrix with nonzero diagonals is in the space $\operatorname{span} R_{F}$ if and only if it is a scalar multiple of the matrix of the form

$$
\left(\begin{array}{ccc}
1 & a & b \\
\frac{1}{a} & 1 & \frac{b}{a} \\
\frac{1}{b} & \frac{a}{b} & 1
\end{array}\right)
$$

with nonzero complex numbers $a$ and $b$. Note that the above matrix belongs to $\left(\operatorname{span} R_{F}\right) \backslash R_{F}$ whenever $|a| \neq 1$. Since $\operatorname{span} R_{F^{\tau}}$ is the full matrix algebra, we conclude that the face $F$ is not induced by a face of $\mathbb{T}$ by Theorem 3.2 .

Acknowledgments. Research of the author was partially supported by PARC through KOSEF (NRFK 2009-0093125). The author is grateful to Professor Young-Hoon Kiem for information on subspaces of matrix algebras, and to Professor Marcin Marciniak for valuable conversation during the Workshop.

Added in the proof (July 4, 2012). PPT entangled edge states of $M_{3} \otimes M_{3}$ are completely classified by [49] and [50]. We also refer [51] to further development on the facial structures for separable states.

\section{References}

[1] E. Alfsen, F. Shultz, Unique decompositions, faces, and automorphisms of separable states, J. Math. Phys. 51 (2010), 052201.

[2] W. Arveson, Quantum channels that preserve entanglement, Math. Ann. 343 (2009), 757-771.

[3] I. Bengtsson, K. Życzkowski, Geometry of Quantum States: An Introduction to Quantum Entanglement, Cambridge Univ. Press, Cambridge, 2006. 
[4] C. H. Bennett, D. P. DiVincenzo, T. Mor, P. W. Shor, J. A. Smolin, B. M. Terhal, Unextendible product bases and bound entanglement, Phys. Rev. Lett. 82 (1999), 5385-5388.

[5] D. Bruß, A. Peres, Construction of quantum states with bound entanglement, Phys. Rev. A (3) 61 (2000), 030301.

[6] E.-S. Byeon, S.-H. Kye, Facial structures for positive linear maps in the two dimensional matrix algebra, Positivity 6 (2002), 369-380.

[7] S.-J. Cho, S.-H. Kye, S. G. Lee, Generalized Choi maps in three-dimensional matrix algebras, Linear Algebra Appl. 171 (1992), 213-224.

[8] H.-S. Choi, S.-H. Kye, Facial structures for separable states, J. Korean Math. Soc. 49 (2012), 623-639.

[9] M.-D. Choi, Positive linear maps on $C^{*}$-algebras, Canad. J. Math. 24 (1972), 520-529.

[10] M.-D. Choi, Completely positive linear maps on complex matrices, Linear Algebra and Appl. 10 (1975), 285-290.

[11] M.-D. Choi, Positive semidefinite biquadratic forms, Linear Algebra and Appl. 12 (1975), 95-100.

[12] M.-D. Choi, Positive linear maps, in: Operator Algebras and Applications (Kingston, 1980), Proc. Sympos. Pure Math. 38, Part 2, Amer. Math. Soc., Providence, RI, 1982, $583-590$.

[13] M.-D. Choi, T.-Y. Lam, Extremal positive semidefinite forms, Math. Ann. 231 (1977), $1-18$.

[14] L. Clarisse, Construction of bound entangled edge states with special ranks, Phys. Lett. A 359 (2006), 603-607.

[15] D. P. DiVincenzo, T. Mor, P. W. Shor, J. A. Smolin, B. M. Terhal, Unextendible product bases, uncompletable product bases and bound entanglement, Comm. Math. Phys. 238 (2003), 379-410.

[16] M.-H. Eom, S.-H. Kye, Duality for positive linear maps in matrix algebras, Math. Scand. 86 (2000), 130-142.

[17] K.-C. Ha, Atomic positive linear maps in matrix algebras, Publ. Res. Inst. Math. Sci. 34 (1998), 591-599.

[18] K.-C. Ha, Positive projections onto spin factors, Linear Algebra Appl. 348 (2002), 105-113.

[19] K.-C. Ha, A class of atomic positive linear maps in matrix algebras, Linear Algebra Appl. 359 (2003), 277-290.

[20] K.-C. Ha, Comment on: "Construction of bound entangled edge states with special ranks" [Phys. Lett. A 359 (2006), 603-607] by L. Clarisse, Phys. Lett. A 361 (2007), 515-519.

[21] K.-C. Ha, S.-H. Kye, Construction of entangled states with positive partial transposes based on indecomposable positive linear maps, Phys. Lett. A 325 (2004), 315-323.

[22] K.-C. Ha, S.-H. Kye, Construction of $3 \otimes 3$ entangled edge states with positive partial transposes, J. Phys. A 38 (2005), 9039-9050.

[23] K.-C. Ha, S.-H. Kye, Y. S. Park, Entangled states with positive partial transposes arising from indecomposable positive linear maps, Phys. Lett. A 313 (2003), 163-174.

[24] M. Horodecki, P. Horodecki, R. Horodecki, Separability of mixed states: necessary and sufficient conditions, Phys. Lett. A 223 (1996), 1-8.

[25] M. Horodecki, P. Horodecki, R. Horodecki, Mixed-state entanglement and distillation: is there a "bound" entanglement in nature?, Phys. Rev. Lett. 80 (1998), 5239-5242.

[26] P. Horodecki, Separability criterion and inseparable mixed states with positive partial transposition, Phys. Lett. A 232 (1997), 333-339.

[27] P. Horodecki, M. Lewenstein, G. Vidal and I. Cirac, Operational criterion and constructive checks for the separability of low rank density matrices, Phys. Rev. A (3) 62 (2000), 032310. 
[28] M. Junge, C. Palazuelos, D. Pérez-Garcia, I. Villanueva, M. M. Wolf, Operator space theory: a natural framework for Bell inequalities, Phys. Rev. Lett. 104 (2010), 170405.

[29] H.-J. Kim, S.-H. Kye, Indecomposable extreme positive linear maps in matrix algebras, Bull. London Math. Soc. 26 (1994), 575-581.

[30] W. C. Kim, S.-H. Kye, Extreme rays in $3 \otimes 3$ entangled edge states with positive partial transposes, Phys. Lett. A 369 (2007), 16-22.

[31] K. Kraus, Operations and effects in the Hilbert space formulation of quantum theory, in: Foundations of Quantum Mechanics and Ordered Linear Spaces (Marburg, 1973), Lecture Notes in Phys. 29, Springer, Berlin, 1974, 206-229.

[32] S.-H. Kye, A class of atomic positive maps in 3-dimensional matrix algebras, in: Elementary Operators and Applications (Blaubeuren, 1991), World-Scientific, River Edge, NJ, 1992, 205-209.

[33] S.-H. Kye, Facial structures for positive linear maps between matrix algebras, Canad. Math. Bull. 39 (1996), 74-82.

[34] S.-H. Kye, On the convex set of all completely positive linear maps in matrix algebras, Math. Proc. Cambridge Philos. Soc. 122 (1997), 45-54.

[35] S.-H. Kye, Facial structures for unital positive linear maps in the two dimensional matrix algebra, Linear Algebra Appl. 362 (2003), 57-73.

[36] S.-H. Kye, Facial structures for decomposable positive linear maps in matrix algebras, Positivity 9 (2005), 57-71.

[37] H. Osaka, A class of extremal positive maps in $3 \times 3$ matrix algebras, Publ. Res. Inst. Math. Sci. 28 (1992), 747-756.

[38] A. Peres, Separability criterion for density matrices, Phys. Rev. Lett. 77 (1996), 1413-1415.

[39] A. G. Robertson, Positive projections on $C^{*}$-algebras and extremal positive maps, J. London Math. Soc. (2) 32 (1985), 133-140.

[40] A. Sanpera, D. Bruß, M. Lewenstein, Schmidt number witnesses and bound entanglement, Phys. Rev. A (3) 63 (2001), 050301-050304.

[41] W. F. Stinespring, Positive functions on $C^{*}$-algebras, Proc. Amer. Math. Soc. 6 (1955), 211-216.

[42] E. Størmer, Positive linear maps of operator algebras, Acta Math. 110 (1963), 233-278.

[43] E. Størmer, Decomposable positive maps on $C^{*}$-algebras, Proc. Amer. Math. Soc. 86 (1982), 402-404.

[44] E. Størmer, Separable states and positive maps, J. Funct. Anal. 254 (2008), 2303-2312.

[45] K. Tanahashi, J. Tomiyama, Indecomposable positive maps in matrix algebras, Canad. Math. Bull. 31 (1988), 308-317.

[46] W.-S. Tang, On positive linear maps between matrix algebras, Linear Algebra Appl. 79 (1986), 33-44.

[47] B. M. Terhal, A family of indecomposable positive linear maps based on entangled quantum states, Linear Algebra Appl. 323 (2001), 61-73.

[48] S. L. Woronowicz, Positive maps of low dimensional matrix algebras, Rep. Math. Phys. 10 (1976), 165-183.

[49] Y.-H. Kiem, S.-H. Kye, J. Lee, Existence of product vectors and their partial conjugates in a pair of spaces, J. Math. Phys. 52 (2011), 122201.

[50] S.-H. Kye, H. Osaka, Classification of bi-qutrit positive partial transpose entangled edge states by their ranks, J. Math. Phys. 53 (2012), 052201.

[51] K.-C. Ha, S.-H. Kye, Geometry of the faces for separable states arising from generalized Choi maps, Open Syst. Inf. Dyn. 19 (2012), 1250009. 
